\title{
The role of interstellar filaments in the origin of the stellar initial mass function: Insights from Herschel observations
}

\author{
Philippe André, Vera Könyves, Arabindo Roy \\ and Doris Arzoumanian
}

Laboratoire AIM, CEA/DSM-CNRS-Université Paris Diderot, IRFU / Service d'Astrophysique, C.E. Saclay, Orme des Merisiers, F-91191 Gif-sur-Yvette, France emails: [philippe.andre, vera.konyves, arabindo.roy]@cea.fr, doris.arzou@gmail.com

\begin{abstract}
The origin of the stellar initial mass function (IMF) is one of the most debated issues in astrophysics. Two major features of the IMF are 1) a fairly robust power-law slope at the high-mass end (Salpeter 1955), and 2) a broad peak around $\sim 0.3 M_{\odot}$ corresponding to a characteristic stellar mass scale (cf. Elmegreen et al. 2008). In recent years, the dominant theoretical model proposed to account for these features has been the "gravo-turbulent fragmentation" picture (e.g., Hennebelle \& Chabrier 2008; Hopkins 2012) whereby the properties of interstellar turbulence lead to the Salpeter power law and gravity sets the characteristic mass scale (Jeans mass). We discuss modifications to this picture based on extensive submillimeter continuum imaging observations of nearby molecular clouds with the Herschel Space Observatory which emphasize the importance of filamentary geometry (André et al. 2010; Könyves et al. 2015). The Herschel results point to the key role of the quasi-universal filamentary structure pervading the cold interstellar medium and support a scenario in which star formation occurs in two main steps (cf. André et al. 2014): first, the dissipation of kinetic energy in large-scale turbulent MHD flows generates $\sim 0.1 \mathrm{pc}$-wide filaments (Arzoumanian et al. 2011) in the cold ISM; second, the densest filaments grow and fragment into prestellar cores (and ultimately protostars) by gravitational instability above a critical threshold $\sim 16 M_{\odot} / \mathrm{pc}$ in mass per unit length or $\sim 160 M_{\odot} / \mathrm{pc}^{2}$ in gas surface density $\left(A_{V} \sim 8\right)$. In our observationally-driven scenario, the dense cores making up the peak of the prestellar core mass function (CMF) - likely responsible for the peak of the IMF - result from gravitational fragmentation of filaments near the critical threshold for global gravitational instability. The power-law tail of the CMF/IMF arises from the growth of the Kolmogorov-like power spectrum of initial density fluctuations $\left[P(k) \propto k^{-1.6 \pm 0.3}\right]$ measured along Herschel filaments (Roy et al. 2015), in agreement with the model by Inutsuka (2001), and from the power-law distribution of line masses observed for supercritical filaments.
\end{abstract}

\section{References}

André, Ph., Di Francesco, J., Ward-Thompson, D., et al. 2014, in: H. Beuther et al. (eds.), Protostars and Planets VI (Tucson: University of Arizona Press), p. 27

André, Ph., Men'shchikov, A., Bontemps, S., et al. 2010, A\&̈A, 518, L102

Arzoumanian, D., André, Ph., Didelon, P., et al. 2011, A\& A, 529, L6

Elmegreen, B. G., Klessen, R. S., Wilson, C. D., et al. 2008, ApJ, 681, 365

Hennebelle, P. \& Chabrier, G. 2008, ApJ, 684, 395

Hopkins, P. F. 2012, MNRAS, 423, 2037

Inutsuka, S. 2001, ApJ, 559, L149

Könyves, V., André, Ph., Men'shchikov, A., et al. 2015, A\&A, in press (arXiv:1507.05926)

Roy, A., André, Ph., Arzoumanian, D., et al. 2015, A\&AA, in press (arXiv:1509.01819)

Salpeter, E. E. 1955, ApJ, 121, 161 